The Astrophysical Journal, 276:533-543, 1984 January 15

(C) 1984. The American Astronomical Society. All rights reserved. Printed in U.S.A.

\title{
4.6 MICRON ABSORPTION FEATURES DUE TO SOLID PHASE CO AND CYANO GROUP MOLECULES TOWARD COMPACT INFRARED SOURCES
}

\author{
J. H. Lacy, ${ }^{1}$ F. Baas, ${ }^{2}$ L. J. Allamandola, ${ }^{2}$ S. E. Persson, ${ }^{3}$ P. J. McGregor, ${ }^{3}$ \\ Carol J. Lonsdale, ${ }^{4}$ T. R. Geballe, ${ }^{5}$ and C. E. P. van de Bult ${ }^{2}$ \\ Received 1983 February 22; accepted 1983 June 28
}

\begin{abstract}
Spectra at a resolving power of 840 have been obtained for seven compact "protostellar" sources in the region of the $4.67 \mu \mathrm{m}$ fundamental vibrational band of carbon monoxide. The deep absorption feature seen by Soifer et al. in W33A near $4.61 \mu \mathrm{m}$ is shown to consist of three features: a strong broad feature at $2165 \mathrm{~cm}^{-1}$, a sharp spike at $2140 \mathrm{~cm}^{-1}$, and a "wing" at $2135 \mathrm{~cm}^{-1}$. The same three features are seen in other sources, but with varying relative strengths. Laboratory experiments involving UV irradiation and temperature cycling of "dirty ice" ( $\left.\mathrm{CO}: \mathrm{H}_{2} \mathrm{O}: \mathrm{CH}_{3} \mathrm{OH}: \mathrm{NH}_{3}\right)$ and $\mathrm{CO}: \mathrm{NH}_{3}$ samples allow conclusive identification of the $2140 \mathrm{~cm}^{-1}$ and its associated "wing" feature (at $2135 \mathrm{~cm}^{-1}$ ) with solid CO, and CO complexed to other molecules. The broad $2165 \mathrm{~cm}^{-1}$ feature is due to one or more molecular species containing $\mathrm{C} \equiv \mathrm{N}$ bonds. These species were formed in the laboratory sample by ultraviolet photolysis of the frozen gas mixtures, a process that should also occur in grain mantles irradiated by ultraviolet light. The molecules containing the cyano groups have a much lower vapor pressure than pure frozen $\mathrm{CO}$, and so can survive in considerably warmer environments. Toward W33A the column density of carbon in frozen molecules is comparable to that in the gas phase.

The formation and location of the CO- and CN-bearing grain mantles and sources of UV radiation in cold molecular clouds are discussed. Plausible sources of UV light can produce the observed cyano group features, but only under conditions in which local heat sources do not cause evaporation of the $\mathrm{CO}$ molecules before they can be photoprocessed.

Subject headings: infrared: spectra - interstellar: grains — interstellar: molecules — line identifications molecular processes - stars: pre-main-sequence
\end{abstract}

\section{INTRODUCTION}

Infrared absorption features, attributed to molecules in interstellar grains and grain mantles, have been seen toward many obscured infrared sources (Aitken 1981). The most prominent and most positively identified features are those at $3.1 \mu \mathrm{m}$ and $9.7 \mu \mathrm{m}$, attributed to water ice and amorphous silicates, respectively. Weaker features, more tentatively identified, are seen frequently at $6.0 \mu \mathrm{m}$ and $6.8 \mu \mathrm{m}$. Although the $9.7 \mu \mathrm{m}$ band is seen both in emission and absorption, the others mentioned here are seen only in absorption and only through dense, and presumably cold, molecular clouds. This fact has been interpreted to mean that the water ice and unidentified features are due to relatively volatile molecules in grain mantles (see Hagen, Allamandola, and Greenberg 1980 and references therein). Models of grain mantles, including condensation, evaporation, and photochemistry have been extensively discussed by, for example, Hagen, Allamandola, and Greenberg (1979) and Greenberg (1976).

A relatively weak absorption feature at $4.61 \mu \mathrm{m}$ has been seen toward the bright compact infrared source W33A by Soifer

\footnotetext{
${ }^{1}$ Palomar Observatory, California Institute of Technology, now at University of California, Berkeley.

${ }^{2}$ Laboratory Astrophysics, Huygens Laboratorium, Rijksuniversiteit te Leiden, The Netherlands.

${ }^{3}$ Mount Wilson and Las Campanas Observatories of the Carnegie Institution of Washington.

${ }^{4}$ Department of Astronomy, University of California, Los Angeles.

${ }^{5}$ Kapteyn Astronomical Institute, Rijksuniversiteit te Groningen, The Netherlands; and United Kingdom Infrared Telescope.
}

et al. (1979). They tentatively identified this feature with solid $\mathrm{CO}$ but noted that the vibrational band center of gaseous or pure solid $\mathrm{CO}$ is at $4.67 \mu \mathrm{m}$. Their resolution $(\lambda / \Delta \lambda \sim 70)$ was insufficient to determine the shape of the band. These observations and the possibility of measuring the $\mathrm{CO}_{\text {gas }} / \mathrm{CO}_{\text {solid }}$ ratio by infrared spectroscopy were discussed by Hagen, Allamandola, and Greenberg (1980).

Leger (1983) has recently calculated the fraction of CO in molecular clouds expected to be bound in grain mantles. Although the desorption mechanisms are not fully understood, his results indicate that significant fractions of the $\mathrm{CO}$ in the inner parts of molecular clouds could be in the solid form. Thus radio observations of gaseous $\mathrm{CO}$, which are used extensively to map the distribution of molecular material, may substantially underestimate the $\mathrm{CO}$, and hence $\mathrm{H}_{2}$, column density through massive molecular clouds. If $\mathrm{CO}$ is bound in grains in large quantities, its reactions with atoms and free radicals in photoprocessed grains could play an important role in the chemistry of molecular clouds, in particular the production of high-mass molecules (Hagen, Allamandola, and Greenberg 1979).

We have obtained $4.55-4.73 \mu \mathrm{m}$ spectra of W33A and six other compact molecular cloud sources with much higher resolving power, $\lambda / \Delta \lambda \sim 840$, in order to resolve the feature detected by Soifer et al. (1979) and thus to attempt an identification. In our spectra the $4.61 \mu \mathrm{m}$ band splits into three features which vary in relative strength from source to source. We compare the source spectra with laboratory spectra obtained specifically to study the spectral signatures of simple 
molecular "ices" whose composition is chosen to mimic interstellar grain mantles. These comparisons strongly support the identification with solid $\mathrm{CO}$, demonstrate the presence of the cyano group, $\mathrm{C} \equiv \mathrm{N}$, and show that the variations in relative band strengths can be explained in terms of variations in the composition, the amount of ultraviolet irradiation, and the temperature of the material. We discuss the astrophysical conditions under which the molecules responsible for the observed features are present in grain mantles and compare the optical depths of the solid state molecules to those of the other features and the column densities to those of gaseous CO. As UV irradiation appears to be effective in producing the observed $\mathrm{C} \equiv \mathrm{N}$ group molecules, we discuss possible sources of UV light within molecular clouds.

\section{OBSERVATIONS}

The sources observed, W33A, GL 2591, NGC 7538/IRS 1, NGC 7538/IRS 9, M8E, W51/IRS 2, and W3/IRS 5, were chosen to provide a wide range in $\tau_{9.7}, \tau_{3.1}$, and $\tau_{9.7} / \tau_{3.1}$, and all sources show substantial extinction. These sources are compact, bright infrared objects, thought to be "protostars" although this terminology is certainly ambiguous, as discussed by Wynn-Williams (1982). The data were obtained with a 1-5 $\mu \mathrm{m}$ grating spectrometer, described by Persson, Geballe, and Baas (1982), mounted on the Palomar $5 \mathrm{~m}$ Hale Telescope on the nights of 1982 June 7 and July 2-4. The spectral resolution was $0.0055 \mu \mathrm{m}\left(2.6 \mathrm{~cm}^{-1}\right)$ which corresponds to a resolving power of 840 at $4.65 \mu \mathrm{m}$. Standard infrared chopping and beam switching techniques were used with a focal plane aperture $4^{\prime \prime}$ in diameter and a chopper throw of $20^{\prime \prime} \mathrm{N}-\mathrm{S}$. For the primary data set, 73 point spectra were measured; the sampling interval was 0.46 times the spectral resolution, giving a coverage of 33.7 resolution elements between 4.55 and 4.73 $\mu \mathrm{m}$.

In order to reduce noise caused by seeing and uncertainties in the guiding, a large number of individual grating scans were co-added in a microcomputer which also controlled the position of the grating. The wavelength calibration was determined by observation of standard lamp lines in various orders of the grating spectrometer and was checked using atmospheric absorption features.

The spectral region covered is rather heavily plagued by telluric $\mathrm{H}_{2} \mathrm{O}$ and $\mathrm{CO}$ lines, so the standard stars used to calibrate the spectra were measured as close in the sky and in air mass to the objects as possible. Intermediate spectral type (A and F) standards were used to avoid complications due to stellar $\mathrm{CO}$, and their $4.8 \mu \mathrm{m}$ broad-band magnitudes were used for flux calibration. Corrections resulting from the small differences in air mass between the standards and objects were found for each spectral point by assuming that the standards were featureless over the region measured. These corrections are completely reliable only if the atmospheric features are either unsaturated or spectrally resolved, and the source features are spectrally resolved. Although the broad source features (see below) are adequately resolved, the atmospheric features are not, and small systematic errors at the frequencies of the gaseous $\mathrm{CO}$ lines may be present.

The resulting flux-calibrated spectra are displayed in Figure 1. In most cases our flux densities agree both in magnitude and slope, to within our overall calibration uncertainty of $20 \%$, with the lower spectral resolution results of Soifer et al. (1979) and Willner et al. (1982), although our fluxes tend to be somewhat lower than theirs. The discrepancies probably result from flux lost due to guiding errors or seeing.

A typical standard star spectrum, calibrated by comparison with another standard, is shown in the lower right-hand panel of Figure 1. Here $\alpha$ Cyg was calibrated using $\beta$ Cas, with a difference in air mass between the two data sets of 0.20 . For the seven program objects, the corresponding difference in airmass was 0.06 on average, with a maximum difference of 0.16 for M8E. Several other such star-star comparisons were made, with similarly good results. We therefore feel confident in the reliability of the air-mass corrections, at least for the spectrally resolved features.

The noise levels in the spectra arise from (background) photon statistics plus systematic effects due to the atmosphere. For the brighter sources integration times were made sufficiently long to ensure that the noise was not dominated by photon statistics. A reasonable measure of the noise is simply the pixel-to-pixel variation in each spectrum except for GL 2591, and possibly M8E, where lines of the fundamental vibration-rotation band of gaseous $\mathrm{CO}$ appear to be present.

Emission features at the position of $\operatorname{Pf} \beta$ are evident in the spectra of W3/IRS 5, NGC 7538/IRS 1, W51/IRS 2, and GL 2591. The large apparent strengths of this line in GL 2591 and W3/IRS 5 are difficult to reconcile with the upper limits to the $\operatorname{Br} \alpha$ flux of these objects (Simon, Simon, and Joyce 1979). In fact it is probable that the apparent $\operatorname{Pf} \beta$ emission features arise wholly or in part from flux calibration difficulties. The choice of standards having weak or absent $\mathrm{CO}$ absorption results unavoidably in uncertainties at the $\operatorname{Pf} \beta$ line frequency. We estimate that the equivalent widths of $\operatorname{Pf} \beta$ in the standards are a few angstroms; this could account for the apparent $\operatorname{Pf} \beta$ emission features in the source spectra.

\section{RESULTS}

\section{a) Absorption Features}

The main observational results, apparent in Figure 1, are as follows: (1) The original $4.61 \mu \mathrm{m}$ feature seen by Soifer $e t$ al. (1979) is well resolved in W33A. It is composed of two partially overlapping absorptions. The higher frequency component is rather broad (FWHM $\sim 25 \mathrm{~cm}^{-1}$ ) and symmetric and is centered at $\sim 2165 \mathrm{~cm}^{-1}(4.62 \mu \mathrm{m})$. The second absorption is much narrower (FWHM $\sim 7 \mathrm{~cm}^{-1}$ ) with a core near 2139 $\mathrm{cm}^{-1}$, and a wing extending to lower frequency. We shall henceforth refer to the core as the $2140 \mathrm{~cm}^{-1}(4.673 \mu \mathrm{m})$ feature. (2) In NGC 7538/IRS 9 the same $2140 \mathrm{~cm}^{-1}$ core and low-frequency wing are again present. The absorption in the $2140 \mathrm{~cm}^{-1}$ core is almost complete, while the broad $2165 \mathrm{~cm}^{-1}$ feature is much weaker. (3) In M8E there is evidence for the broad $2165 \mathrm{~cm}^{-1}$ feature but no convincing evidence for the narrow absorption at $2140 \mathrm{~cm}^{-1}$. (4) In both W3/IRS 5 and NGC $7538 /$ IRS 1 , the $2140 \mathrm{~cm}^{-1}$ core is present but considerably weaker than in W33A, and there is no clear evidence for either the wing on the low-frequency side of the $2140 \mathrm{~cm}^{-1}$ feature or the $2165 \mathrm{~cm}^{-1}$ feature. (5) There is no convincing evidence for either feature in GL 2591 or W51/IRS 2, but in GL 2591 and possibly in M8E there is gas phase CO absorption. An identification scale for the $P$ - and $R$-branches of the fundamental $\mathrm{CO}$ absorption band in this region is shown in Figure 1. Puetter et al. (1979) detected a broad 10\%-15\% 


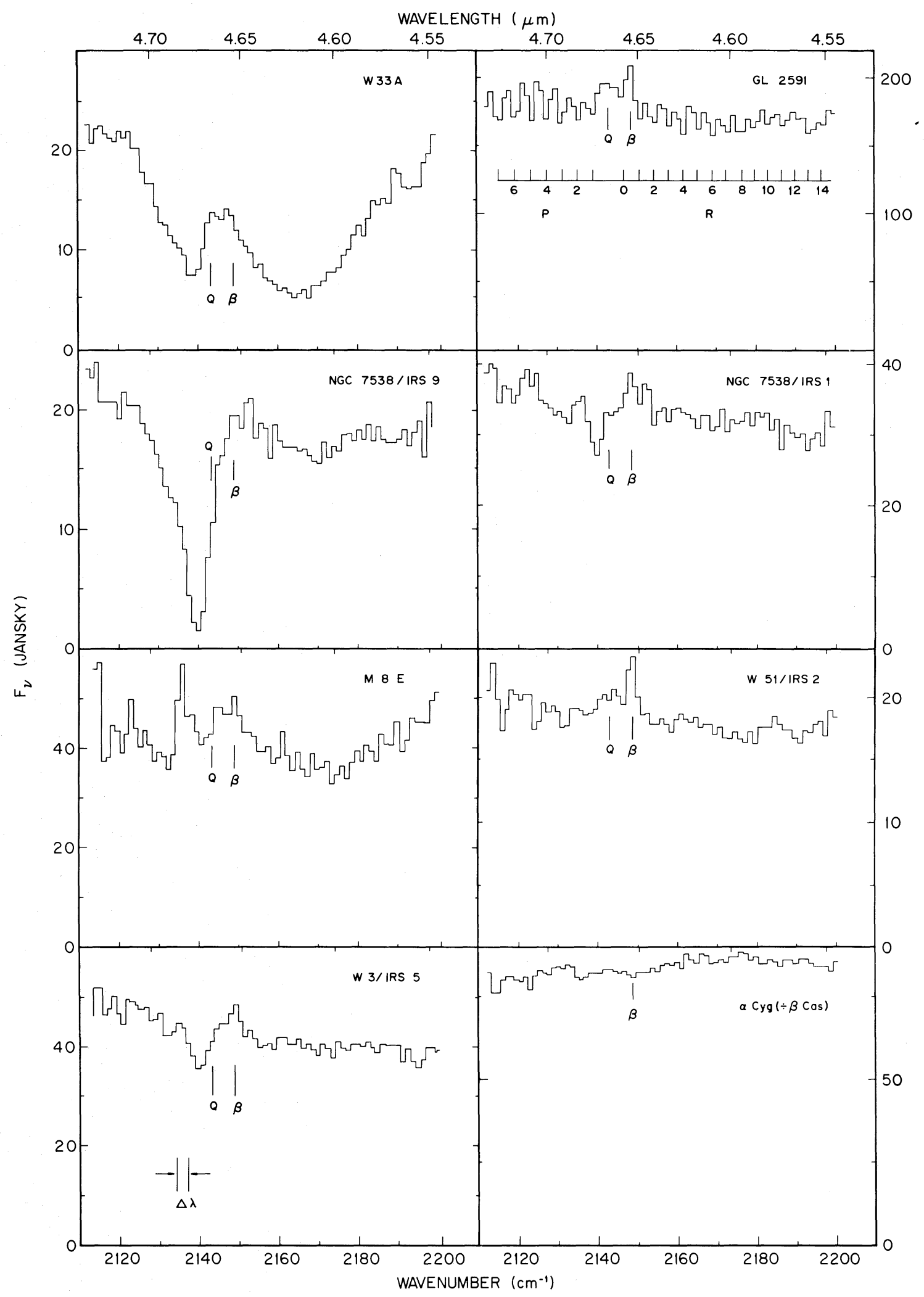

FIG. 1.-Spectra of seven compact sources in the $4.6 \mu \mathrm{m}$ region. The spectral resolution is $\Delta \lambda=0.0055 \mu \mathrm{m}$. $Q$ marks the position of the missing CO $Q$ branch and $\beta$ the position of the Pfund- $\beta$ line. $P$ and $R$ branch lines of gaseous CO are indicated for GL 2591. The bottom right-hand frame demonstrates the reliability of the air-mass correction procedure; see text. 
absorption near $4.7 \mu \mathrm{m}$ in W51/IRS 2, which is not apparent in Figure 1 because our scan covers only the central portion of the absorption. It is probably due to gas phase $\mathrm{CO}$, but higher resolution data are required to establish this with certainty.

In order to examine more closely the structure near 2140 $\mathrm{cm}^{-1}$, a second set of scans of W33A and NGC 7538/IRS 9 was obtained with six rather than two grating steps per resolution element. The resulting spectra, consistent in all respects with the data of Figure 1, are shown in Figure 2; there is no evidence for gas phase $\mathrm{CO}$ absorption in either spectrum. We note a small difference in the wavelength of strongest absorption between W33A and NGC 7538/IRS 9 in both Figures 1 and 2 . The shift amounts to 0.6 resolution elements, or $\sim 1.5 \mathrm{~cm}^{-1}$.

Optical depths and band shapes were obtained from the spectra by assuming that the continua follow straight lines through the highest points, with slopes taken from the spectra of Soifer et al. (1979) and Willner et al. (1982). Because the scanned wavelength interval is only a little wider than the absorption feature itself, the available baseline is not large; some uncertainty in the location of the continuum and in the $\tau$ scale arises from this effect. The spectra of W33A and NGC 7538/IRS 9 are plotted on optical depth scales in Figure 3 . It is noteworthy that the wing on the low-frequency side of the $2140 \mathrm{~cm}^{-1}$ core feature retains much the same strength while both the depth of the core at $2140 \mathrm{~cm}^{-1}$ and the depth of the $2165 \mathrm{~cm}^{-1}$ absorption vary considerably between the two sources. The most natural explanation for this is that there are three independent features - the broad $2165 \mathrm{~cm}^{-1}$ absorp-

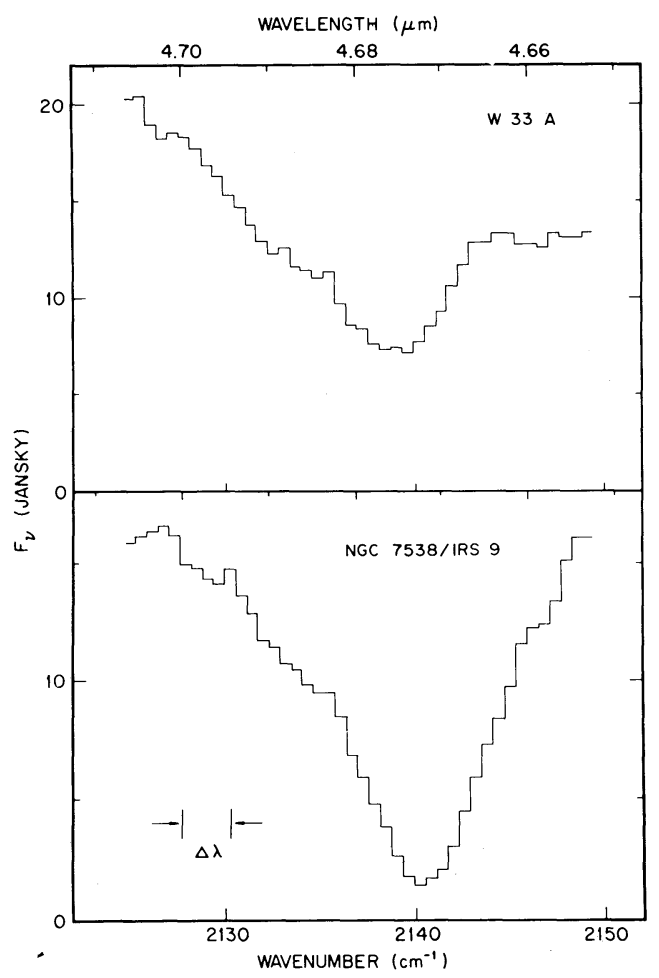

FIG. 2.-Spectra of W33A and NGC $7538 /$ IRS 9 near $2140 \mathrm{~cm}^{-1}$. The spectral resolution is the same as in Fig. 1, but the spectra are more heavily oversampled.

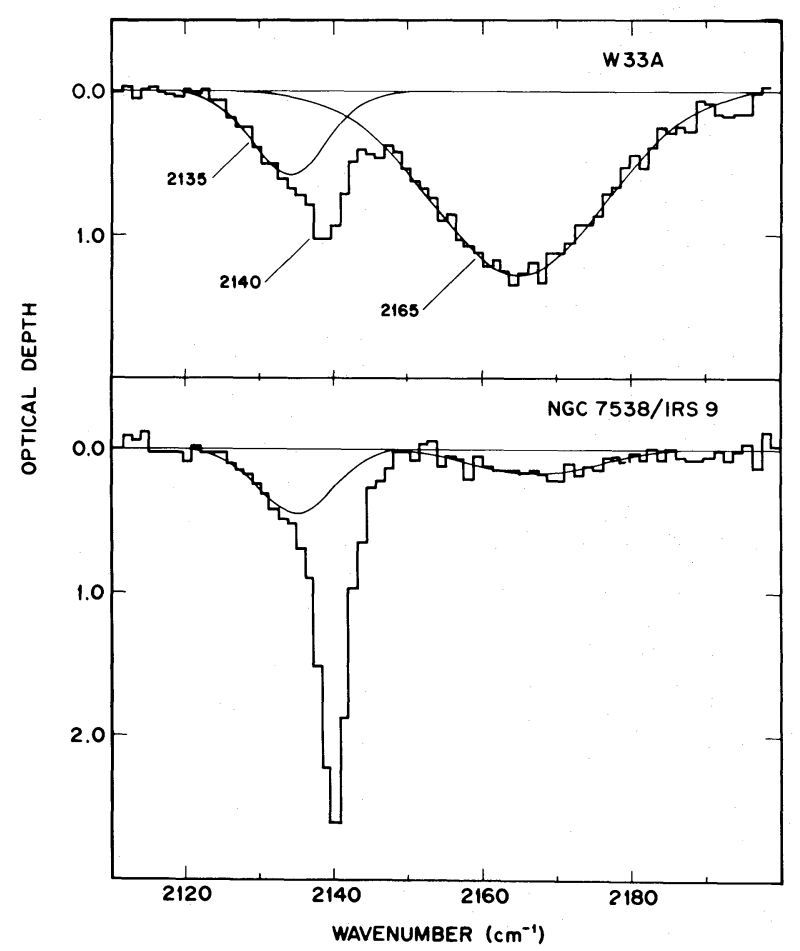

FIG. 3.--Spectra of W33A and NGC 7538/IRS 9 on optical depth scales. The continuous lines are Gaussian fits to the absorption components at $2135 \mathrm{~cm}^{-1}$ and $2165 \mathrm{~cm}^{-1}$; see text.

tion and two superposed absorptions at $2140 \mathrm{~cm}^{-1}$ (the core feature) and $2135 \mathrm{~cm}^{-1}$ (the low frequency wing).

To find characteristic band locations and widths, we obtained multicomponent least squares fits, with the $2140 \mathrm{~cm}^{-1}$ feature in W33A and NGC 7538/IRS 9 modeled as a superposition of two Gaussians separated by $5 \mathrm{~cm}^{-1}$, and the broad $2165 \mathrm{~cm}^{-1}$ feature modeled as a single Gaussian component. While we have no a priori reason to expect any of the features to be Gaussian, the resulting parameters of the fit, given in Table 1, should give accurate relative depths, widths, and locations. Because of uncertainty in the baseline, together with the possibility of a superposition of gaseous $\mathrm{CO}$ and the $2165 \mathrm{~cm}^{-1}$ feature, fits are discussed only for the four sources in which the $2140 \mathrm{~cm}^{-1}$ feature is obvious in Figure 1 . No reliable conclusions could be drawn from the fits to the spectrum of M8E, which is unlike that of any of the others and may contain comparable contributions of gas phase, $2165 \mathrm{~cm}^{-1}$, and $2140 \mathrm{~cm}^{-1}$ features.

In Figure 3 the fits corresponding to the $2165 \mathrm{~cm}^{-1}$ and $2135 \mathrm{~cm}^{-1}$ features are shown (see Table 1). The data are fitted acceptably by the sum of the three Gaussian components used. The decomposition of the $2140 \mathrm{~cm}^{-1}$ feature is not unique, and consequently the width and position of the $2135 \mathrm{~cm}^{-1}$ component are uncertain by several $\mathrm{cm}^{-1}$. The maximum optical depth of the $2140 \mathrm{~cm}^{-1}$ core component is 2.1 in NGC $7538 / \operatorname{IRS} 9$. The $2140 \mathrm{~cm}^{-1}$ core is only marginally resolved in this source but appears to have a width of $\sim 5 \mathrm{~cm}^{-1}$; it would presumably appear somewhat deeper and narrower at higher spectral resolution. It was noted above that the observed location of peak absorption at $2140 \mathrm{~cm}^{-1}$ is different in W33A and NGC 7538/IRS 9. In the fit, the 
TABLE 1

Source Parameters

\begin{tabular}{|c|c|c|c|c|c|c|}
\hline Feature & Parameter & W33A & NGC 7538/IRS 9 & NGC 7538/IRS $1^{a}$ & W3/IRS $5^{\mathrm{a}}$ & Notes $^{b}$ \\
\hline \multirow[t]{5}{*}{$2165 \mathrm{~cm}^{-1}(\mathrm{XCN})$} & $\tau_{\max }$ & 1.28 & 0.16 & $\ldots$ & $\ldots$ & \\
\hline & $v_{0}\left(\mathrm{~cm}^{-1}\right)$ & 2164.8 & 2167.2 & $\cdots$ & $\begin{array}{l}\cdots \\
\cdots\end{array}$ & \\
\hline & $\Delta v(\tau / 2)\left(\mathrm{cm}^{-1}\right)$ & 27.8 & 20.5 & $\ldots$ & $\ldots$ & 1 \\
\hline & $W v\left(\mathrm{~cm}^{-1}\right)$ & 17.5 & 2.6 & $\ldots$ & $\ldots$ & 2 \\
\hline & $\int \tau_{v} d v\left(\mathrm{~cm}^{-1}\right)$ & 37.9 & 3.5 & $\ldots$ & & 3 \\
\hline \multirow[t]{5}{*}{$2140 \mathrm{~cm}^{-1}(\mathrm{CO})$} & $\tau_{\max }$ & 0.56 & 2.09 & 0.24 & 0.20 & \\
\hline & $v_{0}\left(\mathrm{~cm}^{-1}\right)$ & 2139.1 & 2139.9 & 2139.9 & 2140.0 & \\
\hline & $\Delta v(\tau / 2)\left(\mathrm{cm}^{-1}\right)$ & $(5.0)$ & 5.0 & 3.5 & 4.6 & 1,4 \\
\hline & $W v\left(\mathrm{~cm}^{-1}\right)$ & 2.5 & 6.1 & 0.8 & 0.9 & 2 \\
\hline & $\int \tau_{v} d v\left(\mathrm{~cm}^{-1}\right)$ & $(3.0)$ & 11.1 & 0.9 & 1.0 & 3,5 \\
\hline \multirow[t]{5}{*}{$2135 \mathrm{~cm}^{-1}$ (CO, wing) } & $\tau_{\max }$ & 0.58 & 0.46 & $\ldots$ & $\ldots$ & \\
\hline & $v_{0}\left(\mathrm{~cm}^{-1}\right)$ & (2134.1) & 2134.9 & $\ldots$ & $\ldots$ & 4 \\
\hline & $\Delta v(\tau / 2)\left(\mathrm{cm}^{-1}\right)$ & $(12.0)$ & $(12.0)$ & $\ldots$ & $\ldots$ & 1,4 \\
\hline & $W v\left(\mathrm{~cm}^{-1}\right)$ & 6.1 & 4.8 & $\ldots$ & $\ldots$ & 2 \\
\hline & $\int \tau_{v} d v\left(\mathrm{~cm}^{-1}\right)$ & (7.4) & 5.9 & $\ldots$ & $\ldots$ & 3,5 \\
\hline
\end{tabular}

${ }^{\text {a }}$ Fits were not meaningful for the 2135 and $2165 \mathrm{~cm}^{-1}$ features in NGC 7538/IRS 1 or W3/IRS 5.

b NOTES.-(1) Full width of component at $\tau_{\max } / 2$. (2) Equivalent width of single component relative to true continuum. (3) Optical depth of component integrated over frequency. (4) Values in parentheses were not fitted but constrained to be those given. (5) Values in parentheses were derived from constrained data (see note 4).

difference is reduced to only 0.3 times the resolution element because of the different strength of the $2135 \mathrm{~cm}^{-1}$ wing feature. The difference of $2.4 \mathrm{~cm}^{-1}$ in the fitted positions of the broad $2165 \mathrm{~cm}^{-1}$ feature in W33A and NGC 7538/IRS 9 (Table 1) is not likely to be significant, especially when the weakness of the $2165 \mathrm{~cm}^{-1}$ feature in NGC 7538/IRS 9 is considered.

The total equivalent width of the three absorptions in W33A is $32 \mathrm{~cm}^{-1}$, in only fair agreement with the value of $43 \mathrm{~cm}^{-1}$ measured by Soifer et al. (1979). The values of $\tau$ at maximum depth are in agreement when allowance is made for the differing spectral resolutions.

\section{b) Gas Phase CO}

Gas phase CO absorption is clearly present in GL 2591 with absorption lines from $R(0)$ to at least $R(11)$ and $P(1)$ to $P(7)$ being easily identified. A least squares fit with a simplified model of the gaseous $\mathrm{CO}$ band gives an equivalent width of $\sim 0.3 \mathrm{~cm}^{-1}$ for each $\mathrm{CO}$ line, but as no Doppler shift of the band is seen, the Earth's atmosphere must obscure the central $\sim 0.2 \mathrm{~cm}^{-1}$ of each line. Gas must therefore be present with a velocity extent of at least $70 \mathrm{~km} \mathrm{~s}^{-1}$. Gaseous CO absorption also appears to be weakly present in M8E, although the signal-to-noise ratio is not sufficient to allow a reliable identification. Higher resolution spectra are required before any definite conclusions regarding the velocities and rotational temperatures of the CO gas in these two sources can be drawn.

\section{IDENTIFICATION OF THE ABSORPTION FEATURES}

The $4.6 \mu \mathrm{m}$ absorption has been shown to consist of three separate components, centered at $2135 \mathrm{~cm}^{-1}, 2140 \mathrm{~cm}^{-1}$, and $2165 \mathrm{~cm}^{-1}$, whose strengths are largely decoupled from one another. The spectral resolution of the present observations is sufficient to establish that the features seen strongly in W33A and NGC 7538/IRS 9 are not due to gaseous CO. The narrow 2135 and $2140 \mathrm{~cm}^{-1}$ absorptions occur close to the wavelengths of the $P(2)$ and $P(1)$ rotational lines, but there are no accompanying $R(0), R(1)$, and $R(2)$ lines which should be clearly present if all the $\mathrm{CO}$ were in the three lowest rotational levels. The broad $2165 \mathrm{~cm}^{-1}$ feature in W33A and NGC 7538/ IRS 9 cannot be due to a large number of $R$-branch lines because there is no corresponding $P$-branch on the longwavelength side of the $2140 \mathrm{~cm}^{-1}$ feature. Although gaseous $\mathrm{CO}$ absorption may slightly depress the continuum in several of the other sources, it is not significant in W33A and NGC 7538/IRS 9.

The spectral appearance of condensed CO is very different from that of $\mathrm{CO}$ in the gas phase. The $Q$-branch transition, forbidden in the gas phase, is perturbed into activity in a solid environment, while the $P$ - and $R$-branches, which are allowed for gaseous $\mathrm{CO}$, are frozen out in a solid. Furthermore, the central frequency and width of the solid $\mathrm{CO}$ band are dependent on the composition of the solid. Pure solid CO or $\mathrm{CO}$ frozen in an inert matrix has a single narrow absorption (FWHM $\sim 1 \mathrm{~cm}^{-1}$ ) at $2138 \mathrm{~cm}^{-1}$. The line width tends to increase and the central frequency tends to decrease with the addition of impurities. In anticipation of a relation between the source features and solid CO (cf. Hagen, Allamandola, and Greenberg 1980), a specific series of laboratory experiments was carried out in order to attempt identifications of the features.

\section{a) Laboratory Spectra}

In the laboratory experiments simple molecular ices were formed and subjected to conditions similar to those experienced by grain mantles in interstellar space. The general experimental set up has been described by Hagen, Allamandola, and Greenberg (1979). In the present series of experiments, various gaseous mixtures containing $\mathrm{CO}$ were slowly condensed onto an aluminum substrate cooled to $10 \mathrm{~K}$, and $1 \mathrm{~cm}^{-1}$ resolution spectra were taken using a Fourier transform infrared spectrometer. The $\mathrm{CO}$ absorption band was first measured in a mixture consisting of $\mathrm{CO}, \mathrm{H}_{2} \mathrm{O}, \mathrm{CH}_{3} \mathrm{OH}$, and $\mathrm{NH}_{3}$ in the proportions $3: 6: 3: 2$, chosen to approximately reproduce the cosmic abundances of $\mathrm{C}, \mathrm{N}$, and $\mathrm{O}$. The absorption of $\mathrm{CO}$ in this mixture at $10 \mathrm{~K}$ is shown in Figure $4 a$; its maximum occurs at $2138 \pm 1 \mathrm{~cm}^{-1}$. The FWHM of the CO 


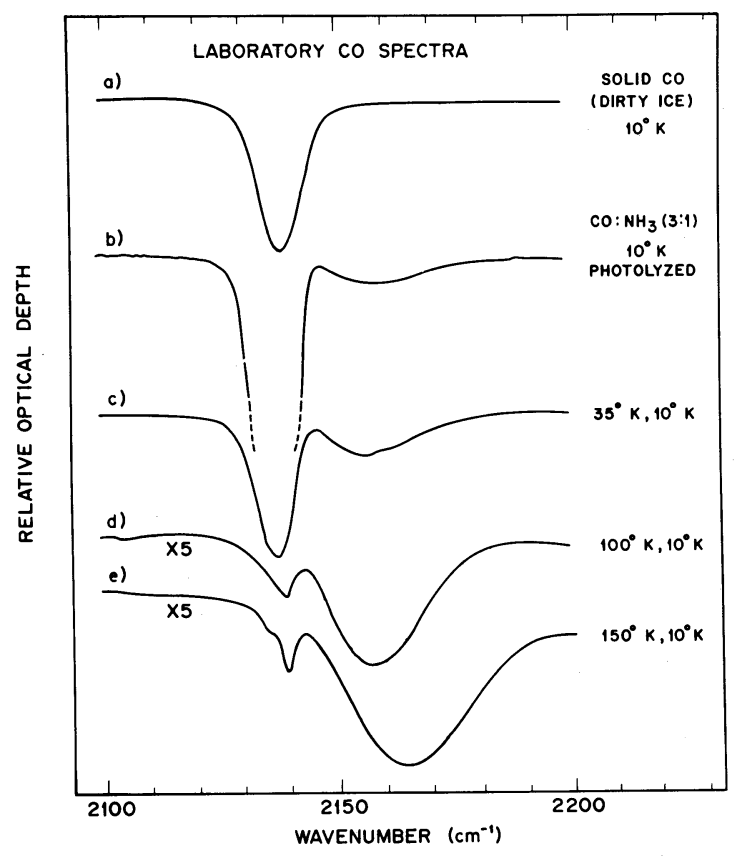

FIG. 4.-Laboratory spectra $\left(1 \mathrm{~cm}^{-1}\right.$ resolution $)$ of some mixtures of simple frozen molecules, including $\mathrm{CO}$, under various conditions of UV-irradiation and temperature cycling. Fig. $4 a$ refers to a mixture of $\mathrm{CO}, \mathrm{H}_{2} \mathrm{O}, \mathrm{CH}_{3} \mathrm{OH}$ and $\mathrm{NH}_{3}(3: 6: 3: 2)$; Figs. $4 b-4 e$ all refer to a mixture of $\mathrm{CO}$ and $\mathrm{NH}_{3}(3: 1)$. The two temperatures given for each trace are, first, those to which the sample was raised, and second, the temperature of the sample at which the spectrum was then measured.

feature has been broadened from $\sim 1 \mathrm{~cm}^{-1}$ (pure solid $\mathrm{CO}$ ) to $11 \mathrm{~cm}^{-1}$ in this "dirty ice."

Next, a sample consisting of $\mathrm{CO}$ and $\mathrm{NH}_{3}$ (3:1) was prepared; the $10 \mathrm{~K}$ spectrum is not significantly different from that of the dirty ice mixture. The $\mathrm{CO}: \mathrm{NH}_{3}$ sample was then irradiated with ultraviolet light from a microwave-powered $\mathrm{H}_{2}$ discharge lamp. Such irradiation induces chemical changes in the deposited sample, by processes which simulate under laboratory conditions the chemical evolution of a grain mantle in the interstellar radiation field. ${ }^{6}$ After prolonged photolysis (typically several hours), a broad absorption feature centered at $\sim 2155 \mathrm{~cm}^{-1}$ was observed (Fig. $4 b$ ) in addition to the original absorption. The explanation for this is that radicals and new molecules have formed during the photolysis.

A series of temperature cycling experiments was then carried out, with the results shown in traces (Figs. $4 c, 4 d$, and $4 e$ ). In each case, the sample was warmed, then cooled to $10 \mathrm{~K}$ and remeasured without further UV irradiation. The warming had two effects: the narrow feature weakened due to evaporation of the $\mathrm{CO}$, and the profile of the broad feature changed somewhat as a result of diffusion-controled reactions between the trapped radicals. Figure $4 e$ shows that when the temperature was cycled to $150 \mathrm{~K}$, the broad feature was dominant and was shifted in frequency to $\sim 2165 \mathrm{~cm}^{-1}$. Note that Figures $4 d$ and $4 e$ are drawn expanded by a factor of 5 , and thus the depth of the broad feature did not change significantly in going

\footnotetext{
${ }^{6}$ The UV photon flux in the laboratory set up, some $10^{7}$ times higher than that in the interstellar medium, does no more than speed up the chemical reaction processes. This and related points are discussed in detail by Hagen, Allamandola, and Greenberg (1979).
}

from $35 \mathrm{~K}$ to $150 \mathrm{~K}$. A weak $\mathrm{CO}$ absorption was still evident in the spectrum at $150 \mathrm{~K}$, presumably because some of the $\mathrm{CO}$ molecules were trapped by larger, less volatile molecules which formed during the photolysis and warm-up. ${ }^{7}$

Several sets of spectra of "dirty ice" were also obtained; these samples were subjected to photolysis times and temperature ranges comparable to those for $\mathrm{CO}: \mathrm{NH}_{3}$. The same features were present in these spectra at similar frequencies.

In order to facilitate the identification of the broad 2165 $\mathrm{cm}^{-1}$ band, three additional binary mixtures, $\mathrm{CO}: \mathrm{CH}_{4}$, $\mathrm{CO}: \mathrm{H}_{2} \mathrm{O}$, and $\mathrm{NH}_{3}: \mathrm{CH}_{4}$, were studied in a manner similar to $\mathrm{CO}: \mathrm{NH}_{3}$. In these experiments the $2165 \mathrm{~cm}^{-1}$ band was observed only in $\mathrm{NH}_{3}: \mathrm{CH}_{4}$ where it was weak. In the $\mathrm{CO}: \mathrm{NH}_{3}$ experiments, the mole fractions studied were 100:1, 50:1, 10:1, $5: 1,1: 1$, and $1: 5 ;$ the formation of the $2165 \mathrm{~cm}^{-1}$ band occurred only in mixtures with an $\mathrm{NH}_{3}$ content in excess of $10 \%$. These results strongly suggest that a molecule containing $\mathrm{C}$ and $\mathrm{N}$ is responsible, and since the cyano group $(\mathrm{C} \equiv \mathrm{N})$ possesses its characteristic fundamental vibrational band in the 2100-2200 $\mathrm{cm}^{-1}$ wavenumber interval (Schimanouchi 1972; Jesson and Thompson 1958), we believe that this absorption indicates the presence of $\mathrm{C} \equiv \mathrm{N}$ groups in one or more molecules which we shall refer to as $\mathrm{XCN}$.

The variation in the relative strengths of the 2140 and $2165 \mathrm{~cm}^{-1}$ features in the temperature cycling experiments (the $2140 \mathrm{~cm}^{-1} \mathrm{CO}$ feature disappears at higher $T$; the 2165 $\mathrm{cm}^{-1}$ feature strengthens slightly with $T$ ) leads to the conclusion that $\mathrm{XCN}$ has a vapor pressure significantly lower than that of pure $\mathrm{CO}$, or even $\mathrm{CO}: \mathrm{NH}_{3}$ complexes. Furthermore, it is significant that a rather high temperature of $150 \mathrm{~K}$ was needed to shift the central absorption to $2165 \mathrm{~cm}^{-1}$; only $30-40 \mathrm{~K}$ should be required for the diffusion and reaction processes. At this stage of the experiments, little more can be said about the identity of XCN. It is anticipated that the results of further isotopic substitution experiments will at least limit the number of possible candidates (van de Bult 1984).

\section{b) Identifications}

Figure 5 shows the comparison between the laboratory spectrum of the photolyzed sample (identical to Fig. $4 e$ ) and the observed spectrum of W33A. The close similarity implies that the essential chemical constituents are present in the laboratory sample. The match of the narrow absorption at $\sim 2140 \mathrm{~cm}^{-1}$ allows an identification of this feature with solid $\mathrm{CO}$ without further discussion. The broad features at 2165 $\mathrm{cm}^{-1}$ in the source and laboratory spectra closely resemble each other and are likely to be due to cyano groups present in stable molecules, as discussed above. The requirement that nitrogen be present in the grains to produce $\mathrm{XCN}$ is not inconsistent with astrophysical constraints; the broad feature also appeared in dirty ice mixtures having cosmic abundances. Also, work by Hagen, Allamandola, and Greenberg (1980) and more recently by Knacke et al. (1982), has shown that $\mathrm{NH}_{3}$ ice can alter the shape of the $3.1 \mu \mathrm{m} \mathrm{H}_{2} \mathrm{O}$ ice band to resemble that observed in the $\mathrm{BN}$ object and other protostellar objects. The "wing" observed at $2135 \mathrm{~cm}^{-1}$ is similar to the wing in the laboratory spectrum and thus is likely to be the remnant of an original absorption which arises from $\mathrm{CO}$

\footnotetext{
${ }^{7}$ It is not certain that the residual pure $\mathrm{CO}$ feature is due to trapped molecules rather than to a small amount of $\mathrm{CO}$ recondensed during cooling.
} 


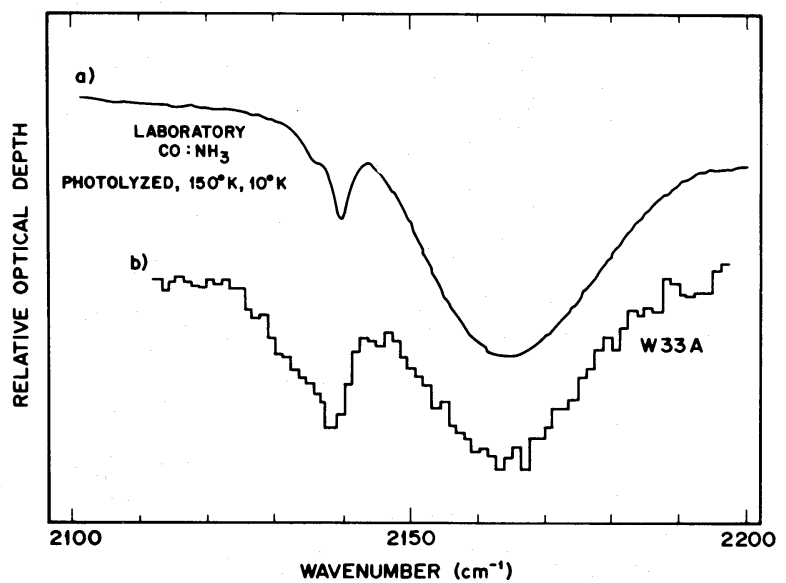

FIG. 5.- The final laboratory spectra of photolyzed $\mathrm{CO}: \mathrm{NH}_{3}$ which has been cycled through $150 \mathrm{~K}$ (same as Fig. 4e), compared with the spectrum of W33A. The optical depth scale is arbitrary.

perturbed by other molecules, such as $\mathrm{NH}_{3}$, in the ice. Lowfrequency wings produced by scattering effects when the wavelength approaches the grain dimensions are another possibility for this feature but require unreasonably large grain diameters of $\geq 1 \mu \mathrm{m}$. The lack of a correlation of the $2135 \mathrm{~cm}^{-1}$ wing feature and $2140 \mathrm{~cm}^{-1}$ solid CO feature between W33A and NGC 7538/IRS 9 also argues against this possibility. Note that the FWHMs of the observed narrow absorption and wing lie between that of pure solid $\mathrm{CO}\left(\sim 1 \mathrm{~cm}^{-1}\right)$ and that of $\mathrm{CO}$ embedded in a dirty ice $\left(\sim 11 \mathrm{~cm}^{-1}\right)$. The observed width of the $\mathrm{CO}$ features can thus be matched by mixtures whose compositions are intermediate between these two extremes.

\section{COMPARISON WITH OTHER SPECTRAL FEATURES}

\section{a) Other Solid State Features}

Table 2 lists the optical depths of several infrared absorption features for the seven observed sources together with the depths of the $\mathrm{CO}$ and $\mathrm{XCN}$ features. There is no correlation of the depth of the $2140 \mathrm{~cm}^{-1} \mathrm{CO}$ feature with other features, but there is a hint of a relationship between the strength of the $2165 \mathrm{~cm}^{-1} \mathrm{XCN}$ feature and that of the 6.0 and $6.8 \mu \mathrm{m}$ features. Any relationship that might exist is dominated by W33A, where the total column density of material is much larger than in the other sources. Nevertheless, it is the $\mathrm{XCN}$ feature, and not the
CO feature, that reflects this fact. This situation, where the $\mathrm{XCN}$ feature is related to the total column of material, while the $\mathrm{CO}$ feature is not, is consistent with the higher temperatures that can be tolerated by the $\mathrm{XCN}$ molecules before they evaporate. These comparisons thus support the idea, based independently on the laboratory data, that the $\mathrm{XCN}$ feature can be more widespread in the molecular clouds, while the CObearing grains must be located in colder, denser regions. Perhaps these cold regions are left over from the collapse of the condensations which formed the infrared objects.

\section{b) Absorption Coefficients and Column Densities}

Absolute infrared vibrational band intensities and cross sections have been determined for solid films of $\mathrm{CO}$ and for $\mathrm{CO}$ frozen in various matrices. The absorption strengths measured were found to be almost identical for $\mathrm{CO}$ monomers, $\mathrm{CO}$ polymers, and $\mathrm{CO}: \mathrm{H}_{2} \mathrm{O}$ complexes (Person 1981). Consequently, the strength of the fundamental $\mathrm{CO}$ stretching mode is not very sensitive to its local environment, and the optical depths of the $\mathrm{CO}$ features can be used to derive column densities for solid CO. The column densities listed in Table 3 were obtained by integrating the observed absorption features (in optical depth plots) over frequency and are based on the frequency-averaged solid $\mathrm{CO}$ absorption band strengths measured by Jiang, Person, and Brown (1975). The entries for the broad $2165 \mathrm{~cm}^{-1} \mathrm{XCN}$ feature were computed under the conservative assumption that the cross section for solid cyano groups is the same as that for $\mathrm{CO}$. The cyano group absorption cross section is in general smaller than the $\mathrm{CO}$ cross section by a factor of 5 or more, and the total column density for solid carbon, frozen in $\mathrm{CO}$ and $\mathrm{XCN}$, is therefore considerably underestimated in Table 3 for W33A. However, without a more specific assignment for the $2165 \mathrm{~cm}^{-1}$ band, the actual XCN column density is difficult to estimate since the cyano group band intensity appears to be rather susceptible to structural factors (Jesson and Thompson 1958).

A determination of the fraction of $\mathrm{CO}$ bound to grains could be made most directly by comparing the column densities determined from the solid state features with the column density derived from gaseous $\mathrm{CO}$ absorption toward the infrared sources. This is not possible with the present data because the spectral resolution is insufficient to detect gaseous $\mathrm{CO}$ features in most of the sources. We instead compare the solid state feature column densities with $\mathrm{CO}$ column densities

TABLE 2

Optical Depths of Infrared Absorption Features

\begin{tabular}{|c|c|c|c|c|c|c|c|c|c|c|}
\hline \multirow[b]{2}{*}{ SOURCE } & \multicolumn{2}{|c|}{$\tau_{3.08}$} & \multicolumn{2}{|c|}{$\tau_{9.7}$} & \multicolumn{2}{|c|}{$\tau_{6.0}$} & \multicolumn{2}{|c|}{$\tau_{6.8}$} & \multirow[b]{2}{*}{$\tau_{\mathrm{XCN}}^{\mathrm{a}}$} & \multirow[b]{2}{*}{$\tau_{\mathrm{CO}^{\mathrm{a}}}$} \\
\hline & Value & Ref. & Value & Ref. & Value & Ref. & Value & Ref. & & \\
\hline W3/IRS 5 & 3.00 & 1 & 7.64 & 1 & 0.33 & 1 & 0.28 & 1 & $<0.1$ & 0.35 \\
\hline M8E ....... & 0.18 & 1 & 2.6 & 1 & 0.00 & 1 & 0.12 & 1 & $<0.2^{\mathrm{b}}$ & $<0.1^{\mathrm{b}}$ \\
\hline GL $2591 \ldots \ldots \ldots \ldots$ & 0.69 & 1 & 4.14 & 1 & 0.12 & 1 & 0.17 & 1 & $<0.05$ & $<0.05$ \\
\hline NGC $7538 /$ IRS $1 \ldots \ldots \ldots$ & 1.29 & 1 & 6.38 & 1 & 0.37 & 1 & 0.43 & 1 & $<0.1$ & 0.3 \\
\hline NGC $7538 /$ IRS $9 \ldots \ldots \ldots \ldots \ldots$ & 3.28 & 1 & 4.46 & 1 & 0.45 & 1 & 0.36 & 1 & 0.2 & 2.6 \\
\hline $\mathrm{W} 33 \mathrm{~A} \ldots \ldots \ldots \ldots \ldots \ldots \ldots \ldots \ldots$ & $>5.4$ & 1 & 7.84 & 1 & 1.75 & 1 & 1.49 & 1 & 1.3 & 1.0 \\
\hline W51/IRS $2 \ldots \ldots \ldots \ldots \ldots \ldots \ldots$ & 0.8 & 2 & 2.8 & 3 & 0.31 & 4 & 0.39 & 4 & $<0.1$ & $<0.1$ \\
\hline
\end{tabular}

a Apparent optical depths at $2165 \mathrm{~cm}^{-1}\left(\tau_{\mathrm{XCN}}\right)$ and $2140 \mathrm{~cm}^{-1}\left(\tau_{\mathrm{CO}}\right)$.

${ }^{\mathrm{b}}$ Likely due to gaseous $\mathrm{CO}$.

REFERENCES.-(1) Willner et al. 1982. (2) Soifer, Russell, and Merrill 1976. (3) Gillett et al. 1975. (4) Puetter et al. 1979. 
TABLE 3

Column Densities

\begin{tabular}{crccrc}
\hline \hline Species & W33A & NGC 7538/IRS 9 & NGC 7538/IRS 1 & W3/IRS 5 & Notes $^{\mathrm{a}}$ \\
\hline$N\left(\mathrm{CO}_{\text {solid }}\right) \ldots \ldots \ldots \ldots$. & 1.0 & 1.8 & 0.2 & 0.2 & 1,2 \\
$N\left(\mathrm{XCN}_{\text {solid }}\right) \ldots \ldots \ldots \ldots .$. & 3.6 & $>0.4$ & $\ldots$ & $>0.1$ & 1,3 \\
$N\left(\mathrm{CO}_{\text {gas }}\right) \ldots \ldots \ldots \ldots \ldots$ & 2.7 & 5.0 & 7.0 & 11.0 & 1,4 \\
$N\left(\mathrm{CO}_{\text {gas }}\right) \ldots \ldots \ldots \ldots \ldots$ & 11.0 & 6.0 & 9.0 & 10.0 & 1,5 \\
\hline
\end{tabular}

${ }^{\text {a }}$ Notes.-(1) In units of $10^{18} \mathrm{~cm}^{-2}$. (2) Assuming $\sigma\left(\mathrm{CO}_{\text {solid }}\right)=1.06 \times 10^{-17} \mathrm{~cm}^{2}$ and including

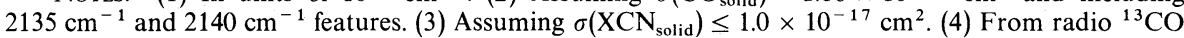
observations; W33A (Goldsmith and Mao 1983; $\Delta v=25-45 \mathrm{~km} \mathrm{~s}^{-1}$ ), NGC 7538 (Dickel, Dickel, and Wilson 1981), W3 (Dickel 1980). (5) From $\tau_{9.7}$ assuming $N(\mathrm{CO}) / \tau_{9.7}=1.3 \times 10^{18} \mathrm{~cm}^{-2}$.

derived from the $9.7 \mu \mathrm{m}$ silicate band optical depths, $\tau_{9.7}$ in Table 2, and from radio ${ }^{13} \mathrm{CO}$ observations. Deriving $\mathrm{CO}$ column densities from $\tau_{9.7}$ requires a knowledge of the ratios $A_{v} / \tau_{9.7}$ and $N(\mathrm{CO}) / A_{v}$, each of which is uncertain by a factor $\sim 2$. We take $A_{v} / \tau_{9.7}=15$ (Gillett et al. 1975) and $N(\mathrm{CO}) / A_{v}=$ $9 \times 10^{16}$ (Frerking, Langer, and Wilson 1982). This method estimates the total $\mathrm{CO}$ column density since $\tau_{9.7}$ carries no information about the fraction of solid phase CO. Column densities of $\mathrm{CO}$ so derived are listed in Table 3. The comparison with radio observations is uncertain for two reasons. First, the radio data sample gas both in front of and behind the infrared sources, and second, some fraction of the absorbing grains could lie in dense clouds closely associated with the infrared sources. In the first case the gaseous $\mathrm{CO}$ column densities would be overestimated, while in the second case they would be underestimated because small dense clouds would not contribute significantly to the ${ }^{13} \mathrm{CO}$ emission.

Dickel, Dickel, and Wilson (1981) have studied the ${ }^{13} \mathrm{CO}$ emission from the NGC 7538 region in some detail; their values of $N\left({ }^{13} \mathrm{CO}\right)$ calculated under LTE assumptions are $\sim 6 \times 10^{16}$ $\mathrm{cm}^{-2}$ toward IRS 9 and $\sim 8 \times 10^{16} \mathrm{~cm}^{-2}$ toward IRS 1 . Taking $N\left({ }^{12} \mathrm{CO}\right) / N\left({ }^{13} \mathrm{CO}\right)=90$, we find $N\left({ }^{12} \mathrm{CO}_{\mathrm{gas}}\right)=$ $5 \times 10^{18} \mathrm{~cm}^{-2}$ toward IRS 9 , and $\sim 7 \times 10^{18} \mathrm{~cm}^{-2}$ toward IRS 1 . The observed silicate feature depths imply very similar values of $N(\mathrm{CO}) \sim 6 \times 10^{18} \mathrm{~cm}^{-2}$ and $9 \times 10^{18} \mathrm{~cm}^{-2}$ for these two sources. In comparison, we derive $N\left(\mathrm{CO}_{\text {solid }}\right) \sim 1.8 \times 10^{18}$ $\mathrm{cm}^{-2}$ and $\sim 0.2 \times 10^{18} \mathrm{~cm}^{-2}$ toward IRS 9 and IRS 1, respectively, from our spectra. $N\left(\mathrm{XCN}_{\text {solid }}\right) \gtrsim 0.4 \times 10^{18} \mathrm{~cm}^{-2}$ toward IRS 9, but with large uncertainty as discussed above. Thus a significant, although probably not dominant, amount of CO is frozen out along the line of sight to NGC 7538/IRS 9, whereas most of the CO remains gaseous toward IRS 1. As indicated by the entries in Table 3, W3/IRS 5 is another source for which the solid phase $\mathrm{CO}$ column density is smaller than the gas phase column density.

For W33A, estimates of the ${ }^{13} \mathrm{CO}$ column density are more uncertain. Goldsmith and Mao (1983) have mapped the entire $\mathrm{W} 33$ region and have found that at the position of $\mathrm{W} 33 \mathrm{~A}$ itself nine distinct velocity components contribute to the ${ }^{13} \mathrm{CO}$ line. Over the interval $25-45 \mathrm{~km} \mathrm{~s}^{-1}, N\left({ }^{13} \mathrm{CO}\right)$ is $\sim 3 \times 10^{16}$ $\mathrm{cm}^{-2}$, indicating $N\left(\mathrm{CO}_{\mathrm{gas}}\right) \sim 2.7 \times 10^{18} \mathrm{~cm}^{-2}$. The present measurements give $N\left(\mathrm{CO}_{\text {solid }}\right) \sim 1.0 \times 10^{18} \mathrm{~cm}^{-2}$ and $N\left(\mathrm{XCN}_{\text {solid }}\right) \gtrsim 3.6 \times 10^{18} \mathrm{~cm}^{-2}$, suggesting that much of the carbon normally found in the gas phase is frozen out. Support for this interpretation comes from the value of $\tau_{9.7}$ which implies $N(\mathrm{CO}) \sim 1 \times 10^{19} \mathrm{~cm}^{-2}$, larger than either the solid or gas phase contributions. The solid $\mathrm{CO}$ column density is probably less than that of gaseous $\mathrm{CO}$, but a substantial fraction of the carbon is likely to be in cyano group molecules. Carbon may also be frozen in grain mantles in other forms. In particular, several carbon-bearing molecules absorb near $6.8 \mu \mathrm{m}$ and may account for the interstellar band at that wavelength. Estimates of column densities of these molecules are not possible at this time, however.

The above comparisons are all quite uncertain but demonstrate that in at least some cases a significant fraction of the $\mathrm{C}$ or $\mathrm{CO}$ in dense molecular clouds is in solid form.

\section{DISCUSSION}

\section{a) Astrophysical Production of the Observed Features}

In the previous sections we have identified the narrow 2140 $\mathrm{cm}^{-1}$ core and $2135 \mathrm{~cm}^{-1}$ features with solid phase $\mathrm{CO}$, perturbed to varying degrees by other solid state molecules, and the broad $2165 \mathrm{~cm}^{-1}$ absorption with solid phase $\mathrm{C} \equiv \mathrm{N}$ bearing molecules $(\mathrm{XCN})$. The presence of the features in laboratory ices containing approximately cosmic abundances of $\mathrm{C}, \mathrm{N}$, and $\mathrm{O}$ support these identifications. The most basic result of the observations and comparison with laboratory spectra is the demonstration that a significant fraction of the $\mathrm{CO}$ molecules can and do condense out of the gas phase in dense molecular clouds.

Leger (1983) has considered theoretically the condensation of $\mathrm{CO}$ molecules onto pure $\mathrm{CO}$-covered dust grains in such clouds and shows, for the case of thermal desorption only, that $\mathrm{CO}$ should be completely frozen at temperatures $\lesssim 17 \mathrm{~K}$ and completely gaseous above that temperature. The present work tends to confirm Leger's suggestion of large solid CO column densities but also demonstrates the existence, in significant amounts, of an additional carbon-bearing, but unidentified, molecule which can be formed from $\mathrm{CO}$, and which has a vapor pressure much lower than that of pure solid $\mathrm{CO}$. Therefore, the temperature of $17 \mathrm{~K}$ derived by Leger for retention of the $\mathrm{CO}$ originally deposited on the CO-covered grains may in fact be too low, and the amount of carbon retained will then depend on how $\mathrm{CO}$ and other organic molecules are produced and destroyed. As a consequence of processes such as those illustrated in the comparison of the laboratory and source spectra above, carbon could remain bound in grain mantles which are subsequently heated far above $17 \mathrm{~K}$. Secondary effects are that CO molecules can stick to the other grain constituents at higher temperatures, and they can be trapped inside an accreted and photoprocessed mantle; these $\mathrm{CO}$ molecules will not evaporate easily. 
The importance of the present results to the question of how general CO depletion on grains might be depends on whether the observed grains are typical of the molecular clouds or are found only in the densest parts of the clouds surrounding the embedded infrared sources. The laboratory temperature cycling experiments, together with the behavior of the relative $\mathrm{CO}$ and XCN optical depths from source to source, provide information on this question and on the astrophysical conditions under which the features should be found. The $2140 \mathrm{~cm}^{-1}$ solid CO feature would be expected mostly in cold, dense clouds, where heating radiation cannot reach the grains, and where reactions with frozen $\mathrm{CO}$ should be negligible. The $2135 \mathrm{~cm}^{-1}$ wing should occur when $\mathrm{CO}$ condenses simultaneously with other molecules. Variations in the relative strengths of the 2135 and $2140 \mathrm{~cm}^{-1}$ features could result from variations in grain temperatures or the composition of the gas.

The laboratory results suggest that the $2165 \mathrm{~cm}^{-1} \mathrm{XCN}$ band results from exposure of simple molecular ices to ultraviolet radiation and temperature cycling. This formation mechanism is discussed in more detail below. Other mechanisms include direct accretion of $\mathrm{XCN}$ from the gas phase and accretion of free radicals which react on grain surfaces to form $\mathrm{XCN}$. It is unlikely that enough XCN molecules could accrete directly, however, as cyano group molecules are much less abundant than $\mathrm{CO}$ in the gas. Cosmic-ray bombardment could also be responsible for the formation of the $2165 \mathrm{~cm}^{-1}$ feature, and in fact absorption features at $2140 \mathrm{~cm}^{-1}$ and $2170 \mathrm{~cm}^{-1}$ have already been seen in laboratory spectra of $\mathrm{H}_{2} \mathrm{O}: \mathrm{NH}_{3}: \mathrm{CH}_{4}$ ices after bombardment by $1 \mathrm{MeV}$ protons (Moore and Donn 1982). UV processing has the advantages that it is efficient, and the UV flux requirements are easily met in regions of the molecular clouds that are not too dense (see below).

A further caution relates to the application of the temperature cycling results to the interpretation of the source spectra. The primary effect of temperature cycling is to increase the diffusion rate of radicals through the sample and hence increase the chemical reaction rate; this begins to occur well below $50 \mathrm{~K}$ in the laboratory experiments. If the astrophysical production of $\mathrm{XCN}$ is governed solely by diffusion-controled reactions in grain mantles, the observed features may be produced at much lower temperatures, such as occur in molecular clouds, over long time scales. On the other hand, the rather high laboratory temperature of $150 \mathrm{~K}$ needed to match the frequency of the XCN feature in the W33A spectrum (Fig. 5) may also be required in the astrophysical case. Quantitative estimates of the temperatures and time scales required to produce $\mathrm{XCN}$ and to provide a good frequency match are not currently available.

\section{b) UV Processing of Grains in Dense Molecular Clouds}

The laboratory experiments show that UV irradiation of a cold $\mathrm{CO}: \mathrm{NH}_{3}$ sample is sufficient to produce a broad absorption feature at $2165 \mathrm{~cm}^{-1}$. With the caveat that competing mechanisms may exist, we now calculate the UV flux required to photoprocess the observed grains and discuss the sources and consequences of UV irradiation. Using the calculations of Hagen, Allamandola, and Greenberg (1979), we estimate that at a flux of $10^{8} \mathrm{UV}$ photons $\mathrm{cm}^{-2} \mathrm{~s}^{-1}$ (typical of the diffuse interstellar medium), the time required for the number of photons absorbed by a grain to equal the total number of molecular bonds in the grain is $\sim 200$ years. After taking into account an estimate for the photolysis efficiency per photon, a correction for the attenuation of UV photons in the deepest part of the grains, and an upper limit for the production of radicals, a time scale of $\sim 10^{4}$ years results. ${ }^{8}$ This corresponds to a total exposure of $3 \times 10^{19} \mathrm{UV}$ photons $\mathrm{cm}^{-2}$ for the complete photoprocessing of grain mantles. What flux of UV photons can be tolerated by the grains without causing them to release their $\mathrm{CO}$ molecules before they can be photoprocessed? At grain temperatures $T_{\mathrm{gr}} \sim 20 \mathrm{~K}$ the wavelength of peak thermal emission far exceeds the grain radius, $a$, and the power emitted by a single grain, according to Dwek et al. (1980), is $L_{\mathrm{gr}}=2.5 \times 10^{-17} a(\mu \mathrm{m})^{4} T_{\mathrm{gr}}{ }^{6}$ ergs s $\mathrm{s}^{-1}$, for the case in which the absorption efficiency $Q(\lambda) \propto \lambda^{-2}$ at long wavelengths. The equilibrium temperature for a grain exposed to (only) a direct source of heating is then obtained by equating $L_{\mathrm{gr}}$ with the energy absorbed from the incident radiation field, $L_{\mathrm{abs}}=\pi a^{2} \alpha\langle Q(\mathrm{UV}) F(\mathrm{UV})\rangle$, where $\alpha \equiv\langle Q(\lambda) F(\lambda)\rangle$ । $\langle Q(\mathrm{UV}) F(\mathrm{UV})\rangle$ is the ratio of the total energy absorbed to the UV energy absorbed and the angle brackets indicate integration over frequency. Setting the UV grain absorption efficiency $Q(\mathrm{UV})=1$ and $F(\mathrm{UV})=1.3 \times 10^{-11} N_{\mathrm{UV}} \operatorname{ergs~s}^{-1}(1500 \AA$ photons), where $N_{\mathrm{UV}}$ is the UV photon flux in photons $\mathrm{cm}^{-2} \mathrm{~s}^{-1}$, and $a=0.1 \mu \mathrm{m}$, we obtain $T_{\mathrm{gr}}=17 \mathrm{~K} \times\left(7 \times 10^{-8}\right.$ $\left.N_{\mathrm{UV}} \alpha\right)^{1 / 6}$. Thus in order that $T_{\mathrm{gr}}$ be less than $17 \mathrm{~K}$, the temperature found by Leger (1983) for complete CO evaporation we require $N_{\mathrm{UV}}<\left(1.4 \times 10^{7}\right) / \alpha$. The value of $\alpha$ depends strongly on the spectral energy distribution of the incident radiation field; for the diffuse interstellar field $\alpha \sim 20$ (Allen 1973), but for a heavily reddened source $\alpha$ can become quite large. If $\alpha=20, N_{\mathrm{UV}}<7 \times 10^{5}$ photons $\mathrm{cm}^{-2} \mathrm{~s}^{-1}$, corresponding to a photoprocessing time scale of $\sim 10^{6}$ years. For heavily reddened sources within molecular clouds $\alpha>20$, the diffuse infrared radiation field will increase $\alpha$ further, and the photoprocessing time scale must therefore be $\gg 10^{6}$ years.

What sources of UV radiation could exist in cold molecular clouds? Until recently, a commonly held belief was that there should be no UV light within dense clouds because of the large extinction. However, there are several ways in which the grains could be exposed to UV light. First, we note that during the transition from the diffuse cloud medium into a denser condensation within a molecular cloud, accretion and photolysis are efficient and the photolyzed grain mantle can be partly built up during the early cloud stage (Greenberg 1976). Also, photolysis will always be important at the outer parts of dense molecular clouds. Boland and de Jong (1982) suggest that cloud material circulates from the deep interior out to the surface and back into the cloud. This process, given enough time, would expose all of the grains to UV light at some point in their lifetimes.

Second, Prasad and Tarafdar (1983) suggest that the interiors of dense clouds may contain a faint steady flux of UV light, which arises ultimately from ionization of $\mathrm{H}_{2}$ molecules by cosmic rays. Ejected electrons excite other $\mathrm{H}_{2}$ molecules into the first electronic state which decays (in the Lyman and Werner bands) to ground. Decays to nonzero vibrational states produce photons which are able to penetrate the cloud

\footnotetext{
${ }^{8}$ The total number of UV photons absorbed in the laboratory experiment is comparable to that derived from the above cloud condensation time scale and photon flux of $10^{8}$ photons $\mathrm{cm}^{-2} \mathrm{~s}^{-1}$.
} 
to a much greater degree than those produced by decays to $v=0$. The mean free paths of the penetrating photons are set by the dust opacity, and the total photon flux is therefore only weakly dependent upon density, $n_{\mathrm{H}_{2}}$. For the Prasad and Tarafdar photon flux of $1350 \mathrm{~cm}^{-2} \mathrm{~s}^{-1}$, a photoprocessing time scale of $\left(3 \times 10^{19}\right) / 1350 \mathrm{~s} \sim 7 \times 10^{8}$ years results; this is longer than the lifetime of molecular clouds. The Prasad and Tarafdar photon flux is a lower limit, and the true value could conceivably meet the photoprocessing requirements. Both of the above mechanisms of exposing grains to UV irradiation would result in photolysis of grains throughout molecular clouds, and XCN might then be ubiquitous.

It is also possible that dense condensations near the infrared sources have been irradiated by local sources of UV light, either the "protostars" themselves, or nearby young stars. Although the mean free path of UV radiation in dense molecular clouds is small, $\sim 10^{16} \mathrm{~cm}$ near W33A, for a ZAMS B0 star at a distance of $10^{16} \mathrm{~cm}$ only $10^{-9}$ of the photons in the range 912-2000 $\AA$ are required to completely photolyze the grains in a time of $10^{6}$ years. The primary problem with this model is that the infrared energy density will far exceed that in the UV, and $\alpha$ will be $\gg 20$. The incident flux must then be low, and this results in a photoprocessing time scale $\gg 10^{6}$ years, which seems unrealistically long. One way out of this problem is to suppose that the value of $17 \mathrm{~K}$ for the $\mathrm{CO}$ evaporation temperature is too low, perhaps because most of the $\mathrm{CO}$ has been trapped by a mantle of XCN molecules produced in an earlier phase of grain mantle evolution. The photoprocessing time scale for the rest of the mantle will then shorten as $\left(17 / T_{\mathrm{gr}}\right)^{6}$.

To summarize, the UV fluxes required for photoprocessing are not particularly stringent. The answer to whether the photoprocessing (and consequent presence of the XCN feature) is a general and widespread process, or a highly local one, is not clear, but the problem of grain heating by local infrared sources in the cloud interiors (i.e., the extended time scales resulting from photoprocessing at low grain temperatures) points to a spatially extended photoprocessing environment, and a long time scale. The weakness of XCN absorption in most of the observed sources would then be due to a small absorption cross section, and its strength relative to the pure $\mathrm{CO}$ absorption feature in $\mathrm{W} 33 \mathrm{~A}$ due to nearly complete evaporation of the pure solid CO.

\section{SUMMARY}

The absorption feature near $4.6 \mu \mathrm{m}$ seen by Soifer et al. (1979) in W33A has been reobserved with a spectral resolving power of 840. Three spectrally resolved features are seen: a broad (FWHM $\sim 25 \mathrm{~cm}^{-1}$ ) feature centered near $2165 \mathrm{~cm}^{-1}$ $(4.62 \mu \mathrm{m})$ with $\tau \sim 1.3$, and two blended absorptions at 2140 $\mathrm{cm}^{-1}$ and $2135 \mathrm{~cm}^{-1}$, each with FWHM $\sim 7 \mathrm{~cm}^{-1}$ and $\tau \sim 0.5$. Six other "protostellar" sources were also observed. NGC 7538/IRS 9 shows the same features as W33A, but with the $2140 \mathrm{~cm}^{-1}$ absorption significantly stronger $(\tau \sim 2.1)$, and the $2165 \mathrm{~cm}^{-1}$ feature weaker. M8E may show only a moderately weak $2165 \mathrm{~cm}^{-1}$ band. W3/IRS 5 and NGC 7538/IRS 1 show moderately weak $2140 \mathrm{~cm}^{-1}$ absorption. Gas phase CO absorption is seen in GL 2591 and probably also in M8E.

Laboratory experiments were carried out to determine the identities of the molecules responsible for the observed bands. "Dirty ice" mixtures of $\mathrm{CO}, \mathrm{H}_{2} \mathrm{O}, \mathrm{CH}_{3} \mathrm{OH}$, and $\mathrm{NH}_{3}$, and also $\mathrm{CO}$ plus $\mathrm{NH}_{3}$ at $10 \mathrm{~K}$ were irradiated with ultraviolet light and then cycled to higher temperatures. The $2140 \mathrm{~cm}^{-1}$ absorption and the $2135 \mathrm{~cm}^{-1}$ wing were found to be due to solid $\mathrm{CO}$ and $\mathrm{CO}$ perturbed by neighboring molecules. An absorption band which reproduces the $2165 \mathrm{~cm}^{-1}$ feature was seen only after irradiation and temperature cycling of samples containing $\mathrm{NH}_{3}$ and $\mathrm{CO}$ or $\mathrm{CH}_{4}$ and is due to molecules containing cyano $(\mathrm{C} \equiv \mathrm{N})$ groups. These $(\mathrm{XCN})$ molecules have much lower vapor pressures than do the original ice constituents.

Comparisons of the source optical depths in solid $\mathrm{CO}$ and $\mathrm{XCN}$ to those of other solid state features support the idea that XCN could be much more widespread than solid $\mathrm{CO}$. Comparisons with radio $\mathrm{CO}$ observations show that significant, although probably not dominant, fractions of the $\mathrm{CO}$ in dense molecular clouds could be frozen on grains. Much of the carbon can remain in heated grain mantles if, prior to heating, it is converted to low vapor pressure compounds like the observed XCN molecules responsible for the 2165 $\mathrm{cm}^{-1}$ absorption.

The sequence of processes carried out in the laboratory are a plausible method of creating the molecules responsible for the $2165 \mathrm{~cm}^{-1}$ feature in astrophysical environments. The ultraviolet radiation and the resulting photolyzed grains may be located in the vicinity of the protostars or may extend throughout the molecular clouds. However, it is likely that at least some fraction of the photoprocessing has taken place away from localized infrared sources.

We are grateful to B. T. Soifer, K. Sellgren, and J. H. Elias for helpful discussions and to K. Y. Lo for allowing us to use some of his telescope time. We thank an anonymous referee for a useful comment. We are grateful to A. Leger, S. S. Prasad, and S. P. Tarafdar for sending their results to us before publication, and Marietta Stevens for efficient preparation of the manuscript. This work was supported in part by the National Science Foundation, the National Aeronautics and Space Administration, and the Netherlands Foundation for the Advancement of Pure Research (Z. W. O.).

\section{REFERENCES}

Aitken, D. K. 1981, in IAU Symposium 96, Infrared Astronomy, ed. C. G Wynn-Williams and D. P. Cruikshank (Dordrecht: Reidel), p. 207. Allen, C. W. 1973, Astrophysical Quantities (London: The Athlone Press). Boland, W., and de Jong, T. 1982, Ap. J., 261, 110.

Dickel, H. R. 1980, Ap. J., 238, 829.

Dickel, H. R., Dickel, J. R., and Wilson, W. J. 1981, Ap. J. (Letters), 250, L43. Dwek, E., Sellgren, K., Soifer, B. T., and Werner, M. W. 1980, Ap. J., 238, 140. Frerking, M. A., Langer, W. D., and Wilson, R. W. 1982, Ap. J., 262, 590.

Gillett, F. C., Forrest, W. J., Merrill, K. M., Capps, R. W., and Soifer, B. T. 1975, Ap. J., 200, 609.
Goldsmith, P. G., and Mao, X. 1983, Ap. J., 265, 791.

Greenberg, J. M. 1976, Ap. Space Sci., 39, 9 .

Hagen, W., Allamandola, L. J., and Greenberg, J. M. 1979, Ap. Space Sci., 65,215 .

. 1980, Astr. Ap., 86, L3.

Jesson, J. P., and Thompson, H. W. 1958, Spectrochimica Acta., 13, 217.

Jiang, G. J., Person, W. B., and Brown, K. G. 1975, J. Chem. Phys., 62, 1201.

Knacke, R. F., McCorkle, S., Puetter, R. C., Erickson, E. F., and Krätschmer, W. 1982, $A p$. J., 260, 141 .

Leger, A. 1983, Astr. Ap., 123, 271. 
Moore, M. H., and Donn, B. 1982, Ap. J. (Letters), 257, L47.

Person, W. B. 1981, in Matrix Isolation Spectroscopy ed. A. J. Barnes, W. J. Orville-Thomas, A. Müller, and R. Gaufrès (Dordrecht: Reidel), p. 415.

Persson, S. E., Geballe, T. R., and Baas, F. 1982, Pub. A.S.P., 94, 381.

Prasad, S. S., and Tarafdar, S. P. 1983, Ap. J., 267, 603.

IOI Puetter, R. C., Russell, R. W., Soifer, B. T., and Willner, S. P. 1979, Ap. J., 228, 118.

Schimanouchi, T. 1972, Tables of Molecular Frequencies, Vol. 1 (National Bureau of Standards Publ. NSRDS-NBS 39).
Simon, T., Simon, M., and Joyce, R. R. 1979, Ap. J., 230, 127.

Soifer, B. T., Puetter, R. C., Russell, R. W., Willner, S. P., Harvey, P. M., and Gillett, F. C. 1979, Ap. J. (Letters), 232, L53.

Soifer, B. T., Russell, R. W., and Merrill, K. M. 1976, Ap. J., 210, 334.

van de Bult, C. E. P. 1984, in preparation.

Werner, M. W., Becklin, E. E., Gatley, I., Matthews, K., and Wynn-Williams, C. G. 1979, M.N.R.A.S., 188, 463

Willner, S. P., et al. 1982, Ap. J., 253, 174

Wynn-Williams, C. G. 1982, Ann. Rev. Astr. Ap., $20,597$.

L. J. Allamandola, F. BaAs, and C. E. P. van De Bult: Laboratory Astrophysics, Huygens Laboratorium, Rijksuniversiteit te Leiden, Wassenaarseweg 78, Leiden, The Netherlands

T. R. Geballe: United Kingdom Infrared Telescope, 900 Leilani Street, Hilo, HI 96720

J. H. LACY: Space Sciences Laboratory, University of California, Berkeley, CA 94720

C. J. Lonsdale: Department of Astronomy, University of California, Los Angeles, CA 90024

P. J. McGregor and S. E. Persson: Mount Wilson and Las Campanas Observatories, Carnegie Institution of Washington, 813 Santa Barbara Street, Pasadena, CA 91101 